\title{
Rate-distortion optimal tree algorithms for piecewise polynomials
}

\author{
Rahul Shukla ${ }^{1}$, Minh N. Do, Pier Luigi Dragotti, and Martin Vetterli \\ Dept. Communication Systems, EPFL, CH-1015 Lausanne, Switzerland \\ email: \{rahul.shukla, minh.do, pierluigi.dragotti, martin.vetterli\}@epfl.ch
}

\section{INTRODUCTION}

Recently, there has been a growing interest in the study of piecewise polynomial functions as an approximation to piecewise smooth functions. Wavelets have long been considered ideal candidates for piecewise smooth function due to their vanishing moment properties. It was shown in $[1,2]$ that for piecewise polynomial signals, the distortion of wavelet based coder decays as $D(R) \sim d_{0} \sqrt{R} 2^{-d_{1} \sqrt{R}}$. But in the same case, it is possible to achieve

$$
D(R) \sim c_{0} 2^{-c_{1} R}
$$

using an oracle method, and this can be realized with a polynomial computational cost using dynamic programming [2]. At low rates, such an algorithm works well for piecewise smooth signals too. The dynamic segmentation algorithm in [2] achieves the right R-D characteristic, but its computational cost is high and it can not be generalized to 2-D.

In this paper, we are interested in a coding scheme, which achieves oracle like asymptotic R-D behavior with polynomial complexity for 1-D as well as 2-D signals. In particular, for the 1 -D case we present a coding scheme which utilizes binary tree segmentation with optimal bit allocation among different segments. Investigation of the algorithm reveals the inherent weakness in the initial coding scheme, leading to a suboptimal performance $\left(D(R) \sim c_{2} \sqrt{R} 2^{-c_{3} \sqrt{R}}\right)$. Reinvestigation of the algorithm also reveals the path to an optimal scheme, which achieves the desired R-D behavior as indicated in eqn.(1).

Most importantly, the optimal binary tree scheme in the 1$D$ case can be easily extended to the $2-D$ case as an optimal quadtree scheme with the similar computational complexity. The proposed optimal quadtree scheme also achieves the oracle like R-D performance for some simple classes of images whereas for the 2-D case there is no known algorithm achieving the right $\mathrm{R}-\mathrm{D}$ behavior at reasonable computational cost.

\section{1-D CASE: BINARY TREE ALGORITHM}

Classical binary tree algorithm: Our algorithm employs a binary tree segmentation followed by a coding algorithm on each signal block in an operational R-D optimal sense. We employ an operational R-D optimization that is similar to the approach used in [3] in finding best wavelet packet bases. A decision strategy based on optimizing R-D performance for each signal block is designed so that the coder can decide if a signal block is worth to be further divided and coded with some appropriate quantization level. The algorithm can be summarized as follows [3]:

1. Segmentation of the input signal: a binary tree segmentation scheme is employed.

2. Optimal representation of each sub-signal by the polynomial model, in a R-D sense.

\footnotetext{
${ }^{1}$ This work was supported by Swiss National Science Foundation Grant 20-63664.00.
}

3. Optimization of this representation to achieve the best reconstructed signal for a given bit rate constraint and distortion measure (MSE). Optimization is performed in R-D sense using Lagrangian cost functional for an optimal quality factor $(\lambda)$.

The binary tree segmentation acts like a singularity detector, so when the tree grows to capture the singularity in a region, it divides the same polynomial piece repeatedly. The classical coding scheme encodes these subdivided pieces of the same polynomial separately. This independent coding of the dependent nodes results in a suboptimal R-D behavior.

Improved binary tree algorithm: The improved coding scheme employs the same previous classical binary tree coding scheme followed by the neighbor joint coding algorithm which, in fact, decides whether neighboring leaves are coded jointly or separately. Thus the neighbor joint coding algorithm is essentially introduced to exploit the inter node dependency. This dependent coding leads to the desired exponentially decaying R-D behavior given by (1).

\section{Conclusion}

Numerical results (Figure 1) indicate that the proposed binary tree based compression algorithm represents a possible way to model and approximate piecewise polynomial signals or, more generally, piecewise smooth signals.

Similar R-D results can also be achieved by the dynamic segmentation algorithm proposed in [2] with the large computational cost $\left(O\left(N^{3}\right)\right)$ whereas our optimal binary tree based compression scheme achieves the desired R-D behavior with much lower computational cost $(O(N \log N))$. While dynamic programming techniques do not work for 2-D problems, our binary tree based coding algorithm can be easily extended to 2-D problems in the form of quadtree based coding

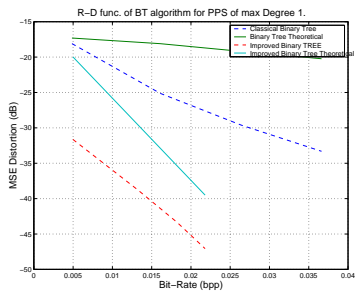

Figure 1: Theoretical(solid) and numerical(dotted) R-D curves for classical and improved binary tree algorithms. algorithm with a reasonable computational complexity. Preliminary simulations show that our quad-tree algorithm outperforms JPEG2000 also in the case of compression of real life images such as Cameraman.

\section{REFERENCES}

[1] A. Cohen, I. Daubechies, O.G. Guleryuz and M.T. Orchard, "On the importance of combining wavelet-based nonlinear approximation with coding strategies," IEEE Trans. Inform. Th., 2000, submitted.

[2] P. Prandoni and M. Vetterli, "Approximation and compression of piecewise smooth functions," Phil. Trans. Royal Society London, August 1999.

[3] K. Ramchandran and M. Vetterli, "Best wavelet packet bases in a rate distortion sense," IEEE Trans. Image Proc., vol. 2, no. 2, pp. 160-175, April 1993. 